\title{
Dietary intake, dietary interventions, nutrient supplements and rheumatoid arthritis: systematic review of the evidence
}

\author{
E. Philippou ${ }^{1}$, S.D. Petersson ${ }^{1}$, S. Erodotou ${ }^{1}$, E. Giallouri ${ }^{1}$, C. Rodomar $^{2}$ and E. Nikiphorou $^{3}$ \\ ${ }^{1}$ Department of Life and Health Sciences, University of Nicosia, 2417, Nicosia, Cyprus, \\ ${ }^{2}$ University of Nicosia Medical School, 2408, Cyprus and \\ ${ }^{3}$ Academic Department of Rheumatology, Division of Immunology, Infection and Inflammatory Disease, King's College \\ London, SE5 9RJ.
}

Rheumatoid arthritis (RA) is a chronic, autoimmune inflammatory disease that continues to cause modest global disability ${ }^{(1)}$. Although the exact aetiopathological factors for disease development are not yet fully understood, it is known that susceptibility to RA is determined by genetic and environmental factors. Poorly controlled RA is associated with fatigue, severe pain and functional disability and possibly 'end-stage' joint damage necessitating surgical intervention ${ }^{(2)}$. Evidence supports that dietary factors or supplements are associated with RA as risk factors for disease onset or triggers for disease flare/progression. We aimed to systematically review the evidence from cohort, case-control studies and clinical trials on diet intake, dietary interventions, fasting and nutrient supplements related to RA risk and outcomes, in order to better understand the role of diet in various aspects of disease.

A systematic search for relevant papers (1946-17 July 2017) was performed on Medline and Embase via the OVID SP platform using the following terms: rheumatoid arthritis or arthritis, rheumatoid or inflammatory arthritis and diet or diet therapy or dietary supplement(s) or dietary intake or nutrition or nutritional supplements or nutrient or nutrients or nutrient uptake or vitamin or vitamin uptake or fasting or diet restriction or "Diet, Food and Nutrition". Studies assessing only intake without relating it to RA risk or outcomes, assessing only serum concentration of micronutrients without reporting intake, studies not published in English, animal studies, abstracts only, editorials and reviews were excluded.

Out of 1952 articles screened, 17 papers included cohort studies, 10 case-control and 68 papers of clinical trials were identified (12 of which were found through hand-searching). Cohort studies assessed dietary factors in relation to the risk of development of RA, these being dietary intake in general $(n=4)$, or specifically intake of protein, meat and iron $(n=1)$, intake of fatty fish and fish oil supplements $(n=1)$, intake of omega-3 fatty acids $(n=1)$, excessive free fructose consumption $(n=2)$, coffee consumption $(n=1)$, antioxidant intake from foods and supplements $(n=2)$ and adherence to the Mediterranean diet $(M D)(n=1)$. Other studies examined dietary factors in relation to disease activity and in particular, general dietary pattern $(n=1)$ and Ramadan fasting $(n=2)$. The relation of vitamin $\mathrm{D}$ and omega-3 fatty acids intake to treatment results $(\mathrm{n}=1)$ was also assessed. Case-control studies assessed diet as a risk in RA development and specifically general dietary factors $(n=2)$, adherence to the MD $(n=1)$, intake of red meat $(n=1)$, intake of alcohol $(n=1)$, omega 3 fatty acids and/or fish and/or fish supplement intake $(n=2)$ and antioxidants $(n=1)$ as well as general diet patterns $(n=1)$. Adherence to the MD in relation to disease activity and treatment outcomes was assessed in one paper. Controlled trials studied the effects of adherence to the MD diet $(n=4)$, vegetarian/vegan $(n=12,5$ of which included a preceding fasting period), elemental $(n=3)$ or peptide diet $(n=1)$, fasting and calorie restriction $(n=11)$ or elimination diets $(n=7)$. 30 papers studied the effects of dietary supplements, namely fish oils/omega-3 or other fatty acids $(n=27)$ and other supplements e.g. Vitamin $E$, Vitamin $K$, Vitamin D alone or in combination with calcium or antioxidants on disease flares $(n=3)$.

In conclusion, from the studies reviewed, dietary factors might be environmental triggers in RA development. In particular, increased meat and excessive free fructose consumption might potentiate RA risk while the MD and fatty fish consumption might reduce RA risk. With regards to disease outcomes, limited periods of fasting reduces disease activity probably by reducing inflammation but this is not a sustainable option. Vegetarian/vegan diets or elimination diets may benefit some patients with undiagnosed food allergies due to diminished immune response to food allergens. Advice thus needs to be individualized. Limited evidence suggests that the MD may benefit pain, swollen and tender joints, and good quality evidence shows that high dose fish oil supplements may improve symptoms and progression of pharmacotherapy. Larger and methodologically-robust randomized controlled trials on dietary interventions are necessary. Due to the heterogeneity of the studies, conclusions on impact and effect size of nutritional interventions is not possible.

1. Cross M, Smith E, Hoy D et al. (2014) Ann Rheum Dis 73:1316-1322.

2. Nikiphorou E, Norton S, Young A et al. (2016) Ann Rheum Dis 75:2080-2086. 